\title{
Diagnose und prädiktive Analysen an zytologischen und bioptischen Tumorproben nicht-kleinzelliger Lungenkarzinome: Aktuelle Strategien und Herausforderungen
}

\author{
Diagnostic and Predictive Analyses of Cytological Specimens of Non-Small Cell Lung Cancer: \\ Strategies and Challenges
}

Autoren

Institute
F. J. F. Herth ${ }^{1}$, L. Bubendorf ${ }^{2}$, S. Gütz ${ }^{3}$, A. Morresi-Hauf $f^{4}$, M. Hummel ${ }^{5}$, K. Junker ${ }^{6}$, U. Lehmann ${ }^{7}$, I. Petersen ${ }^{8}$, P. A. Schnabel ${ }^{9}$, A. Warth ${ }^{10}$

Die Institutsangaben sind am Ende des Beitrags gelistet. $\begin{array}{lll}\text { eingereicht } & \text { 29. } 12.2012\end{array}$ akzeptiert nach Revision 31. 1. 2013

Bibliografie Dol http://dx.doi.org/ 10.1055/s-0032-1326344 Pneumologie 2013; 67: 198-204 (c) Georg Thieme Verlag KG Stuttgart · New York ISSN 0934-8387

Korrespondenzadresse Dr. Arne Warth

Institut für Pathologie Universität Heidelberg Im Neuenheimer Feld 224 69120 Heidelberg arne.warth@med.uniheidelberg.de

\section{Zusammenfassung \\ $\nabla$}

Personalisierte Medizin wird zunehmend zum Standard in der Behandlung des fortgeschrittenen nicht-kleinzelligen Lungenkarzinoms. Tumorspezifische Therapien auf Basis von Biomarkeranalysen, z.B. dem Nachweis von EGFR Mutationen oder Translokationen des $A L K$-Genlokus, übertreffen die undifferenziert eingesetzte Kombinationschemotherapie hinsichtlich des klinischen Ansprechens und des progressionsfreien Überlebens deutlich. Der Nachweis prädiktiver molekularer Alterationen erfordert jedoch eine hohe Kompetenz im Umgang mit Zell- und Gewebeproben. Eine Herausforderung ist hierbei häufig die meist geringe Menge an verfügbarem Untersuchungsmaterial, an welchem sowohl eine spezifische Tumorsubtypisierung als auch ergänzende Biomarkeranalysen durchgeführt werden müssen. Aktuell gibt es nur wenige standardisierte und evidenzbasierte Empfehlungen bezüglich der Probengewinnung, der Materialprozessierung, der Analyse und der Befundung. Entsprechende qualitätsoptimierte Verfahren erfordern eine fachübergreifende Zusammenarbeit, um klinische und pathologische Aspekte gleichermaßen zu berücksichtigen. Um hierfür eine Basis zu schaffen, wurden unterschiedliche Verfahren, Methoden und Protokolle interdisziplinär diskutiert und bewertet. Ein Schwerpunkt lag hierbei neben den unterschiedlichen Verfahren der zytologischen und bioptischen Probengewinnung in der Prozessierung des Materials, um den gestiegenen Anforderungen an Diagnostik und Prädiktion gerecht zu werden. Die dargestellten Einflussgrößen der Probengewinnung und -aufarbeitung sind unter dem Aspekt einer qualitativ hochwertigen und standardisierten Diagnostik von wachsender Bedeutung in der multidisziplinären, zunehmend komplexer werdenden Betreuung von Lungenkrebspatienten.

\section{Abstract \\ $\nabla$}

Personalised medicine is becoming the standard care for advanced non-small cell lung cancer. Tumour-specific therapies based on biomarker analyses, e.g., EGFR mutations or translocations of the $A L K$ gene locus, result in a superior patient outcome compared to unselected therapy approaches. However, predictive molecular analyses can be challenging and require significant experience with cell- and tissue-based diagnostic methods. The major challenge relates to the sometimes low amount of available tumour material for both diagnostic and predictive analyses. As yet, there are no standardised or evidence-based recommendations concerning biopsies, specimen processing, and analyses. Respective guidelines require combined interdisciplinary actions to consider both clinical and pathological aspects. In order to establish a basis for high quality procedures, different approaches, methods, and protocols were interdisciplinary discussed with an emphasis on cytological specimens. Detailed evaluation of the parameters and consented recommendations might contribute to optimised strategies in the interdisciplinary, more and more complex care of non-small cell lung cancer patients. 


\section{Abkürzungsverzeichnis \\ $\nabla$}

ALK anaplastische Lymphomkinase

ATS American Thoracic Society

BAL bronchoalveoläre Lavage

CK Zytokeratin

CT Computertomografie

EUS-FNA endoösophagealer Ultraschall mit Feinnadelaspiration

EBUS endobronchialer Ultraschall

EGFR epidermaler Wachstumsfaktorrezeptor (epidermal growth factor receptor)

ERS European Respiratory Society

FFPE Formalin-fixiertes und in Paraffin eingebettetes (Gewebe)

FISH Fluoreszenz-in-situ-Hybridisierung

IASLC International Association for the Study of Lung Cancer

MGG May-Grünwald-Giemsa

NSCLC nicht-kleinzelliges Lungenkarzinom

PCR Polymerase Kettenreaktion

TBNA transbronchiale Nadelaspiration

TTF-1 thyroidaler Transcriptionsfaktor-1

\section{Einleitung}

Mit jährlich weltweit etwa 1,4 Millionen Todesfällen sind Lungenkarzinome nach wie vor die führende Ursache aller tumorbedingter Sterbefälle [1]. Die historisch gewachsene Einteilung in kleinzellige und nicht-kleinzellige Karzinome muss zunehmend differenzierter betrachtet werden, da jüngste Daten belegen, dass sowohl eine präzise histomorphologische [2] als auch eine immunhistochemische [3] und molekularpathologische [46] Charakterisierung dieser Tumoren einen signifikanten Einfluss auf die Prognose und den Krankheitsverlauf dieser Patienten haben $[7,8]$. So können Patienten mit nicht-kleinzelligen Lungenkarzinomen und aktivierenden EGFR-Mutationen oder Translokationen des ALK-Genlocus [5, 9] im Vergleich zu einer konventionellen Chemotherapie eindeutig von zielgerichteten Therapien mit Tyrosinkinaseinhibitoren profitieren [7, 8,10].

Der Nachweis therapierelevanter Mutationen erfolgt mit unterschiedlichen Methoden unmittelbar am Tumorgewebe selbst und ist im deutschsprachigen Raum flächendeckend in entsprechend qualifizierten Instituten für Pathologie etabliert [11]. Die Standardmethode zum Mutationsnachweis stellt nach wie vor die Sanger Sequenzierung [12] dar, mit welcher potentiell alle EGFR-Mutationen in den untersuchten Exonen nachgewiesen werden können. Einschränkungen ergeben sich jedoch bei der Sensitivität dieser Methode, da für diagnostisch belastbare Ergebnisse ein Tumoranteil von $\sim 30 \%$ im Gewebsschnitt (ohne Mikrodissektion) notwendig ist [5]. Sensitivere Methoden zur Detektion von EGFR-Mutationen sind beschrieben [13-18], finden jedoch in der Routinediagnostik bislang keine regelhafte Anwendung. Ein gewichtiger Faktor zur verlässlichen Analyse prädiktiver Biomarker ist somit die Menge an bioptisch gewonnenem Tumormaterial, welches ausreichend für eine zunehmend detailliertere histomorphologische und (immun-) histochemische Subtypisierung der Tumoren als auch darauf basierenden prädiktiven Biomarkeranalysen sein sollte. Aktuell gibt es nur wenige standardisierte und evidenzbasierte Empfehlungen bezüglich der Materialprozessierung, der Analyse und der Befundung [5, $19,20]$. Eine Verbesserung dieser Situation erfordert interdisziplinäre Konzepte, um von der Art der Probengewinnung bis hin zur Befundinterpretation verlässliche Analysen gewährleisten zu können. Um hierfür eine Basis zu schaffen, wurden fachübergreifend unterschiedliche Vorgehensweisen der Probengewinnung, der Materialprozessierung und der prädiktiven Diagnostik diskutiert und bewertet sowie Konzepte und Strategien für eine qualitätsoptimierte Diagnostik erarbeitet.

\section{Material und Methoden \\ $\nabla$}

Die prädiktive Diagnostik an zytologischen Proben wurde in mehreren Sitzungen der Arbeitsgemeinschaft Pneumopathologie der Deutschen Gesellschaft für Pathologie seit ihrer Gründung im Jahre 2008 [21, 22] ausführlich thematisiert. Unterschiedliche Vorgehensweisen, Methoden und Protokolle wurden diskutiert, unter den Mitgliedern ausgetauscht und validiert. In Vorbereitung auf ein Expertentreffen wurde zusätzlich eine gezielte Literaturrecherche durchgeführt, um bereits publizierte Erfahrungswerte zu integrieren. Relevante Aspekte der Probengewinnung wurden in gemeinsamen Diskussionen mit klinischen Kollegen integriert. Ziel war hierbei, konsentierte Empfehlungen einer qualitätsoptimierten und standardisierten Probengewinnung und Materialprozessierung im Kontext prädiktiver molekularpathologischer Analysen abzugeben.

\section{Ergebnisse \\ $\nabla$}

Aspekte der Probengewinnung für prädiktive

Biomarkeranalysen

Um dem gestiegenen Gewebe- bzw. Zellbedarf in Diagnostik und Prädiktion gerecht zu werden, kann bereits die Wahl der Methode zur Probengewinnung entscheidend sein, z. B. in Abhängigkeit der Tumorlage ( $\bullet$ Abb.1, $\bullet$ Tab. 1). Es wurden daher die unterschiedlichen Möglichkeiten der bioptischen bzw. zytologischen Probengewinnung detailliert diskutiert und bewertet.

Für endoskopisch unmittelbar bzw. durch Kompression indirekt sichtbare Läsionen geeignet ist die transbronchiale Nadelaspiration (TBNA), welche sowohl mit als auch ohne Ultraschall Anwendung findet $[23,24]$. Größere Nadeldurchmesser (z.B. 19G, Innendurchmesser $0,69 \mathrm{~mm}$ ) erzielen hier bessere Ergebnisse, da so in der Regel größere Tumoranteile erfasst werden oder auch Stanzzylinder gewonnen werden können.

Das EBUS-Verfahren (gezielte Probenentnahme mit Hilfe von endobronchialem Ultraschall; EBUS) verbessert die Aussagekraft zytologischer Proben weiter, insbesondere bei der Probengewinnung aus hilären Lymphknoten und Lymphknoten aus dem oberen Mediastinum (Stationen 2 L, 2r, 10-12) [25]. Die Kombination von EBUS-TBNA und EUS-FNA (endoösophagealer Ultraschall mit Feinnadelaspiration) optimiert die diagnostische Aussagekraft in Bezug auf eine Tumordiagnose noch weiter, im besten Fall mit einer Sensitivität von bis zu 96\% und einer Spezifität von $100 \%$ [26].

Bronchiale und transbronchiale Biopsien haben eine hohe diagnostische Wertigkeit, sofern ausreichend große Zangen (offene Zangendurchmesser $\sim 2 \mathrm{~mm}$ ) verwendet und mindestens 4 Proben entnommen werden. Zu kleine Zangen führen zu Artefakten, welche eine spezifische Diagnose mit dem Ziel eines minimalen Gewebeverbrauchs schwierig machen. Bei zu großen Zangen steigt naturgemäß das Komplikationsrisiko [27,28], jedoch in einem vertretbaren Ausmaß. Insbesondere die Methode der Kryobiopsien ist sehr effektiv und sicher, um möglichst große 

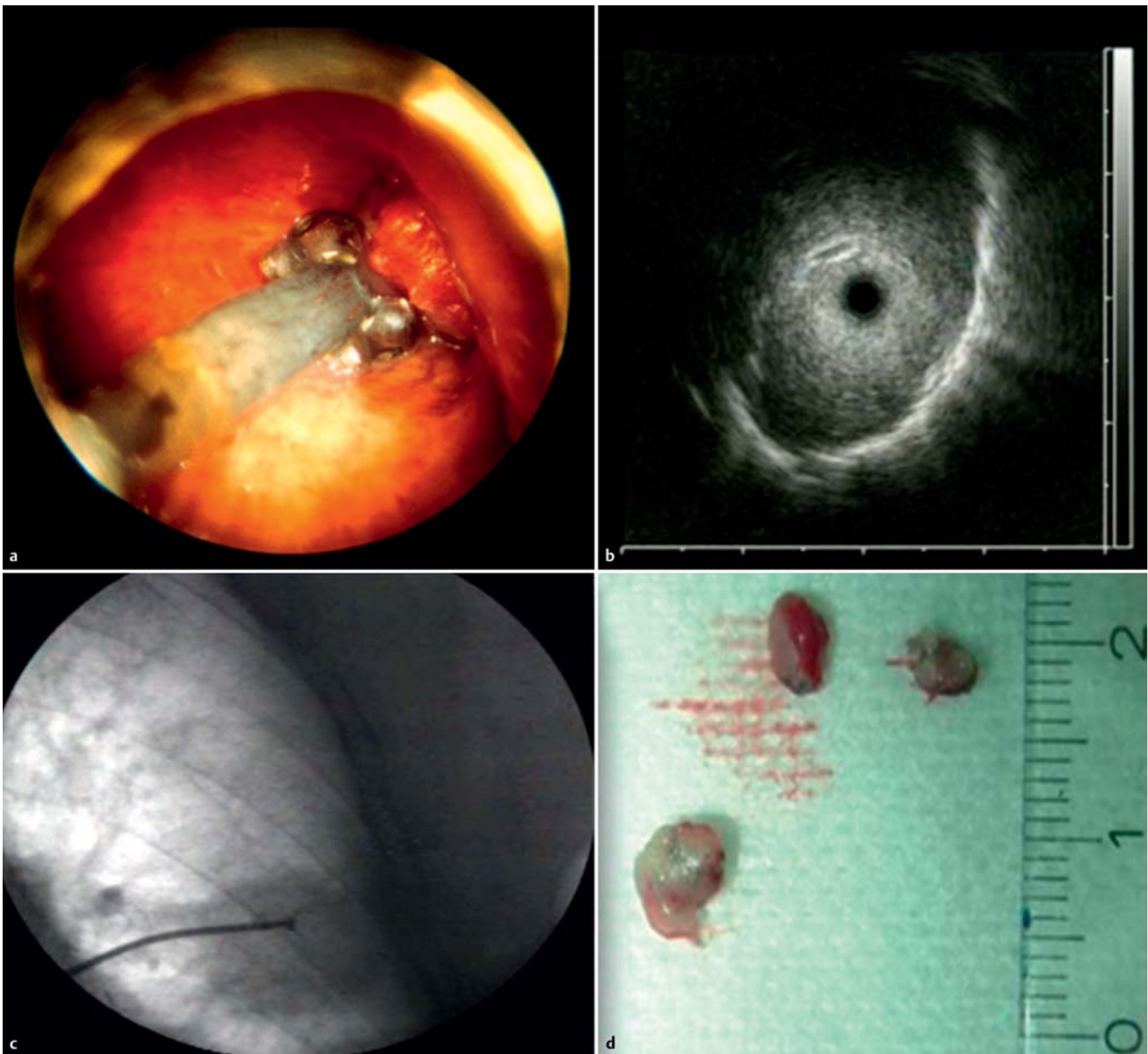

Abb. 1 Für zentrale Tumoren (a) erlaubt der endobronchiale Ultraschall (EBUS; b) eine Steigerung der diagnostischen Effizienz. Für peripher gelegene Tumorherde sind transbronchiale Biopsien unter Durchleuchtung geeignet (c). Abbildung d verdeutlicht den Größenunterschied zwischen einer konventionellen Zangenbiopsie (oben) und einer Kryobiopsie (unten).

Tab. 1 Methoden der Probengewinnung in Abhängigkeit der Tumorgröße und -lage.

\begin{tabular}{|llll|}
\hline Entnahmeverfahren & Material & Klinik & Endoskopischer Befund \\
\hline Bronchoskopie & Sputum & zentrale Lage & endobronchiale/intratumorale Läsion \\
\hline & Zangenbiopsie/ PE-Abstrich & zentrale Lage & endobronchiale/intratumorale Läsion \\
\hline & Tumorpunktion & zentrale Lage & keine sichtbare Läsion \\
\hline & transbronchiale Biopsie & periphere Lage & keine sichtbare Läsion \\
\hline & Bürstenabstrich & periphere Lage & sichtbare Läsion/Zangenbiopsie nicht \\
möglich
\end{tabular}


Gewebeproben zu gewinnen [29]. Diese Methode ist in der transbronchialen Anwendung jedoch noch nicht weit verbreitet und steht aktuell nur in spezialisierten Zentren zur Verfügung.

Transthorakale Nadelbiopsien, meist CT gesteuert, werden häufig eingesetzt, um unklare periphere Lungenrundherde diagnostisch abzuklären. Die Erfolgswahrscheinlichkeit liegt hier bei 80-95\% [30,31]. Der negativ prädiktive Wert des Verfahrens wird mit 84-96\% angegeben, falsch negative Ergebnisse sind lediglich in $2-4 \%$ der Fälle zu erwarten [32]. Hier liegen die Komplikationsraten, meist in Form eines Pneumothorax, bei etwa 20-50\% [33 $-35]$.

Bürstenabstriche können vor oder nach einer Biopsie durchgeführt werden, eine mögliche Kontamination mit Blut infolge einer vorher entnommenen Biopsie scheint keinen signifikanten Einfluss auf die Probenqualität zu haben [36,37]. Die Methode ist insbesondere für endoskopisch sichtbare Tumoren geeignet. Die Bronchiallavage kann ebenfalls vor oder nach einer Biopsie erfolgen, wobei etwa 10-20 ml isotonische Kochsalzlösung empfohlen werden [36-38]. Das gewonnene Material wird in Form von Ausstrichpräparaten und/oder durch die Anfertigung von Zellblöcken aufgearbeitet. Die Methode ist jedoch meist weniger ertragreich als die Bürstenzytologie. Die bronchoalveoläre Lavage (BAL), bei der $3 \times 50 \mathrm{ml}$ isotonische Kochsalzlösung in die peripheren Luftwege eingelassen und wieder aufgefangen werden, wird vor allem für die Diagnostik von infektiösen oder interstitiellen Lungenerkrankungen eingesetzt [39]. Gelegentlich lassen sich damit aber auch Adenokarzinome vom „lepidischen“ Typ diagnostizieren, auch wenn die Methode dafür weniger geeignet ist. Die diagnostische Verwertbarkeit bzw. die Wahrscheinlichkeit einer positiven Tumordiagnostik mittels zytologischer Proben ist bei Sputum am geringsten, gefolgt von Bronchiallavagen, Bürstenabstrichen und Feinnadelaspiraten [20] ( Tab.2). Die Sputumdiagnostik kann aufgrund der geringen Sensitivität [40] nicht empfohlen werden.

Tab. 2 Diagnostische Effizienz in Abhängigkeit unterschiedlicher Entnahmeverfahren.

\begin{tabular}{|l|l|}
\hline Methode & Effizienz (\%) \\
\hline Sputum & $27-41$ \\
\hline Bürstenabstriche & $61-77$ \\
\hline transbronchiale Biopsate (TBB) & $61-77$ \\
\hline bronchoalveoläre Lavage (BAL) & 37,5 \\
\hline Feinnadelaspirat (TBNA/FNA) & 89 \\
\hline
\end{tabular}

Für eine optimale Ausbeute an diagnostisch und prädiktiv verwertbarem Probenmaterial wird empfohlen, unterschiedliche Verfahren wie z.B. Bürstenabstriche und Biopsien bei zentralen Tumoren zu kombinieren $[41,42]$. Mehrere Nadelpassagen (mindestens 3-4 pro suspekter Läsion) erhöhen ebenfalls die Wahrscheinlichkeit für diagnostisch verwertbares Probenmaterial [43].

\section{Aspekte der Zell- und Gewebefixation für prädiktive Analysen}

Nach Entnahme sollte ein etwaiger Transport der Proben keinesfalls gefroren, sondern stets flüssig im Temperaturbereich von $2-8^{\circ} \mathrm{C}$ oder in geeigneten Fixativa (z.B. Alkohol oder gepuffertes Formalin mit definiertem $\mathrm{pH}+$ Pufferkapazität) erfolgen. Bouin'sche Lösung sollte unbedingt vermieden werden, da die enthaltene Pikrinsäure zu DNA-Schäden führt und so molekularpathologische Analysen deutlich erschwert oder unmöglich macht.
Das durch eine BAL gewonnene Zellmaterial sollte in 50\%-70\% Alkohol oder ggf. in gepuffertem Formalin (verdünnt auf $10 \%$ ) fixiert werden, wobei für eine MGG Färbung eine Fixation nicht zwingend erforderlich ist und eine Lufttrocknung bereits ausreicht. Die Fixation von Bürstenabstrichen und TBNA-Material erfolgt in der Regel mit 95\% Alkohol. Um nicht nur Ausstrichpräparate zur Verfügung zu haben, empfiehlt es sich, von zytologischem Material stets Zellblöcke anzufertigen (siehe unten). Bioptisch gewonnenes Gewebe sollte in $10 \%$ gepuffertem Formalin fixiert werden.

\section{Diagnostischer Algorithmus für Zytologiepräparate und Zellblöcke}

Es besteht Konsens, zytologische NSCLC-Proben in Analogie zu dem Schema, welches im Kontext der kürzlich erschienenen IASLC/ATS/ERS-Klassifikation des Adenokarzinoms der Lunge für Biopsien publiziert wurde [44], aufzuarbeiten. Unter Berücksichtigung der Histomorphologie und einer geeigneten Immunmarkerselektion ist so eine verlässliche Biopsiediagnostik bei minimalem Gewebeverbrauch möglich [3].

\section{Allgemeine Aspekte und Protokolle zur Herstellung von Zellblöcken}

Es gibt aktuell keine standardisierten Qualitätskontrollen für molekulare Biomarkeranalysen an Zytologiematerial. Ringversuche in Analogie zu Gewebeproben [11] sind daher wünschenswert, um einen einheitlichen diagnostischen Standard zu gewährleisten.

Die Vorteile von Zellblöcken liegen in der Verfügbarkeit validierter FFPE Protokolle und der Möglichkeit, Serienschnitte anfertigen zu können. Somit kann einerseits das Originalpräparat erhalten bleiben und entsprechend archiviert werden, andererseits können molekulare Analysen ggf. an Folgeschnitten wiederholt werden bzw. bei Tumorprogress weitere prädiktive Biomarker am Archivmaterial analysiert werden ohne die Notwendigkeit einer erneuten Materialentnahme. Zellblöcke ersetzen hierbei keine Zytologie (Ausstrich, Zytospin), sind aber als ergänzendes Verfahren für prädiktive Biomarkeranalysen sehr gut geeignet. Sie bieten weiter den Vorteil einer optimalen Materialausbeute, da die diagnostische Aufarbeitung eines relativ großen Volumens von Zellmaterial möglich und daher ggf. eine bessere Repräsentativität realisierbar ist, z. B. bei TBNAs mit mehreren Nadelpassagen. Grundsätzlich sind alle zytologisch gewonnenen Proben geeignet, um Zellblöcke herzustellen. Das im Folgenden detailliert aufgeführte Gautinger Protokoll ( $\bullet$ Abb.2) kann uneingeschränkt für die molekularzytologische Diagnostik empfohlen werden:

1. Flüssigkeit in ein Zentrifugationsröhrchen abfüllen und 10 Minuten zentrifugieren (4000 U/Min) ( Abb.2a).

2. Den Überstand in ein extra Röhrchen abkippen und aufbewahren.

Es ist empfehlenswert, vom Bodensatz Ausstriche anzufertigen.

3. Auf den Bodensatz 1-2 ml Formalin (10\%ige Lösung; neutral gepuffert) geben. Gut mischen. Mindestens 15 Minuten stehen lassen. Kurz anzentrifugieren (4000 U/Min), den Überstand abkippen $($ Abb.2 b)

4. Einige Tropfen Eiweißglycerin +1,5-2 ml 96\% Alkohol langsam dazu geben und gut mischen $(\bullet \mathbf{A b b} . \mathbf{2 c}$ ). Flocken bilden sich

$(\bullet$ Abb.2d). Das Gemisch für 5 Minuten zentrifugieren (4000 U/Min).

5. Das geronnene Material mit einem Spatel sofort lösen

$(\bullet$ Abb.2e und $\odot$ Abb.2f). 

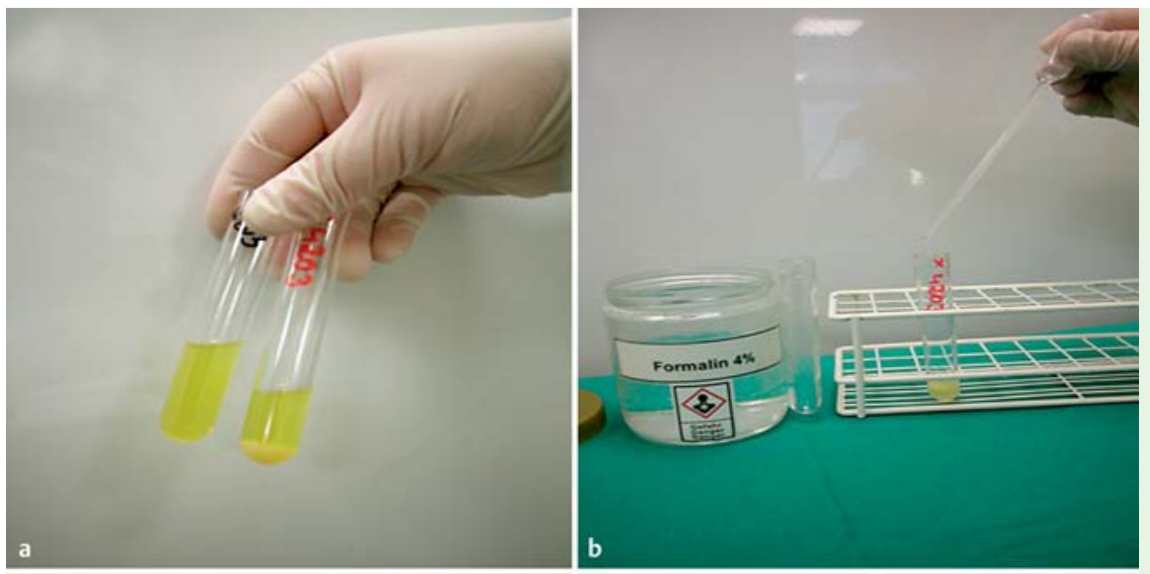

Abb.2 Herstellung eines Zellblockes.

Quelle: Institut für Pathologie, Asklepios Fach-

kliniken München-Gauting.
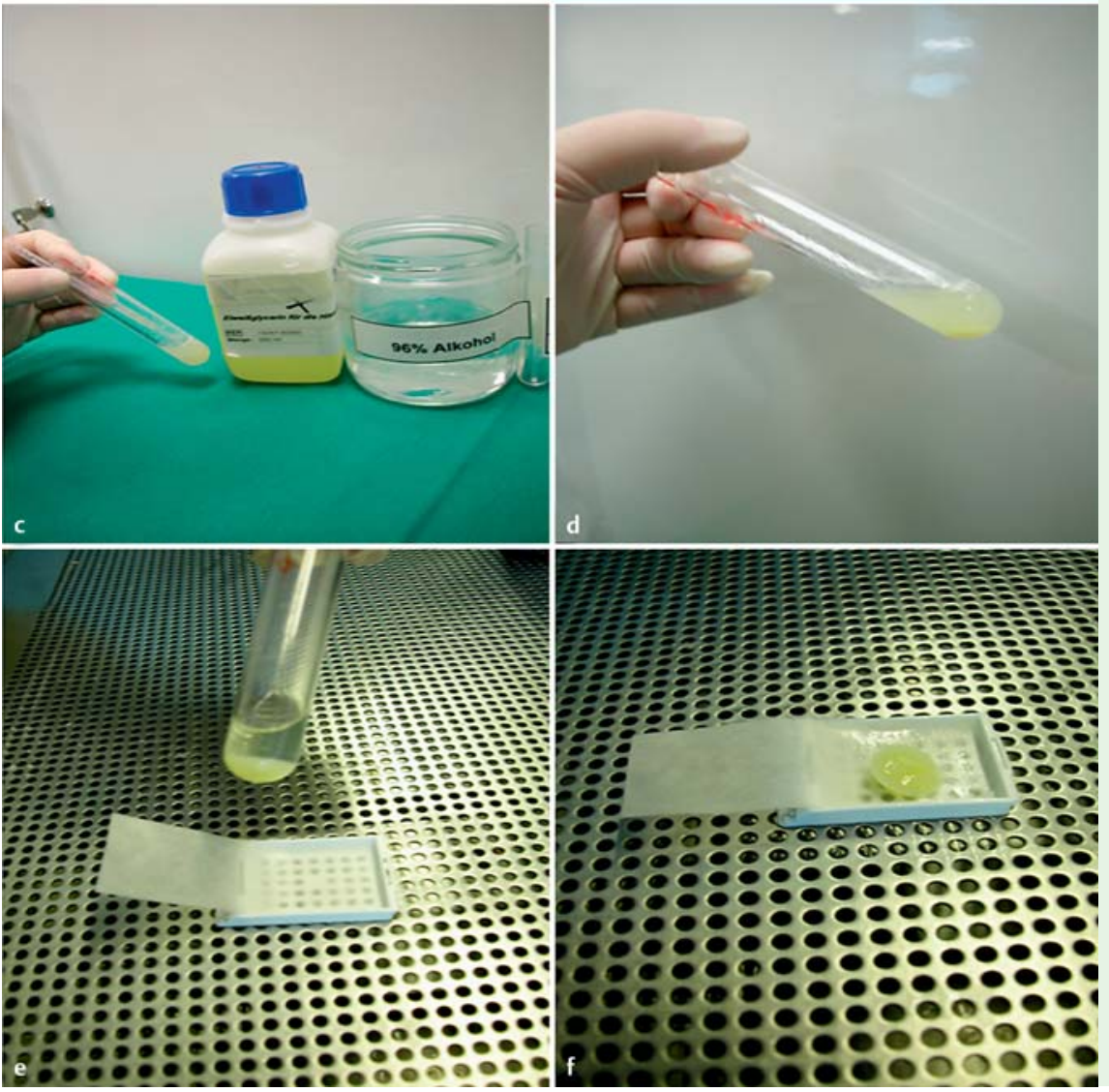

6. Probe in die nummerierte Kassette geben. Weitere Prozessierung analog zu konventionellen Gewebeproben.

Beim Ausgießen (nach der Prozessierung) darauf achten, dass keine vollständig runden Proben, sondern in Stufenschnitten wieder erkennbare Konturen (z.B. im Probenrandbereich) zur Orientierung entstehen. Dies erleichtert eine ggf. folgende Mikrodissektion durch versiertes technisches Personal nach entsprechender Markierung der Objektträger durch den Pathologen. Weiter ist anzumerken, dass die Zugabe von Eiweißglycerin und die mehrfache Zentrifugation nur notwendig sind, wenn sich kein spontanes Koagulat bildet.

Weitere validierte und empfehlenswerte Protokolle zur Herstellung von Zellblöcken werden in Jena [45] und in Basel [46] verwendet.

\section{Diskussion \\ $\nabla$}

Zur Optimierung der prädiktiven Molekulardiagnostik bei nur spärlich vorhandenem Zell- oder Gewebsmaterial nicht-kleinzelliger Lungenkarzinome wurden Verfahren und Protokolle zur Materialprozessierung diskutiert und bewertet. Die Ergebnisse zielen darauf $a b$, eine interdisziplinäre Basis für die qualitätsoptimierte Probengewinnung und die Aufarbeitung von entsprechenden Proben zu schaffen, um so den gestiegenen Anforderungen an Diagnostik und Prädiktion gerecht zu werden.

Zum Zeitpunkt der Erstdiagnose sind mehr als zwei Drittel der Patienten mit nicht-kleinzelligem Lungenkarzinom inoperabel $[47,48]$. In diesen Fällen steht zur Tumortypisierung und zur prädiktiven Diagnostik ausschließlich bioptisches oder zytologisches Material zur Verfügung, was einen rationalen Umgang mit dem Gewebe erfordert $[20,44]$. Bei ca. $40 \%$ aller Patienten erfolgt die Diagnose ausschließlich an zytologischem Material [49]. Gerade 
solche Patienten könnten jedoch von einem positiven prädiktiven Biomarkernachweis profitieren. Insofern sollte uneingeschränkt jedes zur Verfügung stehende Tumormaterial einer solchen Diagnostik unterzogen werden. Bei morphologisch nicht sicher klassifizierbaren NSCLC, welche bis zu einem Drittel aller Fälle ausmachen [3], sollte die immunhistochemische bzw. immunzytochemische Subtypisierung möglichst mit wenigen spezifischen und sensitiven Immunmarkern (TTF-1, p40, p63, CK5/6) erfolgen, um weiteres Material für prädiktive Analysen zur Verfügung zu haben $[3,49]$.

Prinzipiell sind an zytologischem Material bei ausreichendem Tumorgehalt alle gängigen Zusatzuntersuchungen (Immunzytochemie, PCR-basierte Analysen, FISH) ohne Einschränkungen durchführbar, was mit unterschiedlichen Methoden und Kollektiven in der Literatur hinreichend belegt ist [49-56]. Von Vorteil im Vergleich zu bioptisch gewonnenem Tumormaterial kann dabei sogar sein, dass im Präparat häufig weniger nicht-neoplastische Zellen enthalten sind, welche z.B. PCR-basierte DNA-Analysen in ihrer Sensitivität einschränken können [5].

Auf Basis der hier dargelegten Aspekte ergeben sich für eine standardisierte Aufarbeitung von zytologischem oder spärlich bioptisch gewonnenem Material im Hinblick auf prädiktive molekulare Biomarkeranalysen folgende Empfehlungen:

- Sofern im klinischen Kontext vertretbar, sollte stets so viel Tumormaterial wie möglich gewonnen werden.

- Sputumdiagnostik und Bronchiallavage sollten nach Möglichkeit mit Verfahren kombiniert werden, welche eine ausreichende Menge an Tumormaterial gewährleisten (TBNA oder bioptische Verfahren).

- Wenn ein Transport zytologischer Proben notwendig ist, sollte dies flüssig in geeigneten Fixativa oder bei $2-8^{\circ} \mathrm{C}$ erfolgen. Ein Einfrieren der Probe muss vermieden werden.

- Neben Ausstrichen sollte von zytologisch positiven Proben stets ein Zellblock angefertigt werden, da dies molekularpathologische Analysen oftmals erleichtert. Die Zellblockherstellung sollte aus Gründen der Effizienz unmittelbar nach zytologischer Malignitätsdiagnose anhand des bei $4 \mathrm{Grad}$ Celsius aufbewahrten Restsediments erfolgen. Bei allen EBUSTBNA und bei allen klinisch malignitätsverdächtigen Ergüssen sollte dies gleich zu Beginn erfolgen.

- Zytologische und bioptisch gewonnene Proben sollten mit möglichst minimalem Verbrauch von Untersuchungsmaterial aufgearbeitet werden, um weiteres Material für prädiktive Analysen zur Verfügung zu haben. Ein Algorithmus auf Basis der IASLC/ATS/ERS-Klassifikation [44] wird empfohlen.

- An zytologischem und bioptisch gewonnenem Material sind prinzipiell alle prädiktiven Analysen zuverlässig durchführbar. Falls das Biopsiematerial nur eingeschränkt repräsentativ ist oder ausschließlich zytologisches Tumormaterial zur Verfügung steht, sollten prädiktive Biomarkeranalysen an diesem Material durchgeführt werden.

\section{Interessenkonflikt}

Der interdisziplinäre Austausch im Rahmen von Expertentreffen und das Erstellen des Manuskriptes (durch A. Warth) wurden durch AstraZeneca unterstützt.

\section{Fazit für die Praxis}

Zusammenfassend lässt sich festhalten, dass auch spärlich zytologisches oder bioptisch gewonnenes Tumormaterial unter Berücksichtigung der aufgeführten Bedingungen ohne wesentliche Einschränkungen für prädiktive Markeranalysen geeignet ist. Die dargestellten Einflussgrößen der Probengewinnung und -aufarbeitung sind unter dem Aspekt einer qualitativ hochwertigen und standardisierten Diagnostik von wachsender Bedeutung in der multidisziplinären, zunehmend komplexer werdenden Betreuung von Lungenkrebspatienten.

\section{Institute}

${ }^{1}$ Abteilung für Pneumologie und Intensivmedizin, Thoraxklinik am

Universitätsklinikum Heidelberg

2 Institut für Pathologie, Universität Basel

${ }^{3}$ Robert-Koch Klinik, Klinikum St. Georg Leipzig

${ }^{4}$ Institut für Pathologie, Asklepios Fachkliniken München-Gauting

${ }^{5}$ Institut für Pathologie, Charité, Berlin

${ }^{6}$ Zentrum für Pathologie, Klinikum Bremen Mitte

${ }^{7}$ Institut für Pathologie, Medizinische Hochschule Hannover

8 Institut für Pathologie, Universitätsklinikum Friedrich-Schiller-Universität Jena

${ }^{9}$ Sektion Pneumopathologie, Institut für Pathologie, Universität Heidelberg

${ }^{10}$ Institut für Pathologie, Universität Heidelberg

\section{Literatur}

1 Jemal A, Bray F, Center MM et al. Global cancer statistics. CA Cancer J Clin 2011; 61: 69-90

2 Warth A, Muley T, Meister $M$ et al. The novel histologic International Association for the Study of Lung Cancer/American Thoracic Society/ European Respiratory Society classification system of lung adenocarcinoma is a stage-independent predictor of survival. J Clin Oncol 2012; 30: $1438-1446$

3 Warth A, Muley T, Herpel E et al. Large-scale comparative analyses of immunomarkers for diagnostic subtyping of non-small-cell lung cancer biopsies. Histopathology 2012; 61: 1017-1025

$4 \mathrm{Mok}$ TS. Personalized medicine in lung cancer: what we need to know. Nat Rev Clin Oncol 2011; 8: 661 - 668

5 Penzel R, Schirmacher P, Warth A. A Novel EML4-ALK Variant: Exon 6 of EML4 Fused to Exon 19 of ALK. J Thorac Oncol 2012; 7: 1198-1199

6 Warth A, Macher-Goeppinger S, Muley $T$ et al. Clonality of multifocal nonsmall cell lung cancer: implications for staging and therapy. Eur Respir J 2012; 39: 1437 - 1442

7 Kwak EL, Bang YJ, Camidge DR et al. Anaplastic lymphoma kinase inhibition in non-small-cell lung cancer. N Engl J Med 2010; 363: 1693 1703

8 Mok TS, Wu YL, Thongprasert S et al. Gefitinib or carboplatin-paclitaxel in pulmonary adenocarcinoma. N Engl J Med 2009; 361: 947 - 957

9 Soda $M$, Choi YL, Enomoto $M$ et al. Identification of the transforming EML4-ALK fusion gene in non-small-cell lung cancer. Nature 2007; 448: 561 - 566

10 Rosell R, Carcereny E, Gervais $R$ et al. Erlotinib versus standard chemotherapy as first-line treatment for European patients with advanced EGFR mutation-positive non-small-cell lung cancer (EURTAC): a multicentre, open-label, randomised phase 3 trial. Lancet Oncol 2012; 13: $239-246$

11 Penzel R, Sers C, Chen Yet al. EGFR mutation detection in NSCLC-assessment of diagnostic application and recommendations of the German Panel for Mutation Testing in NSCLC. Virchows Arch 2011; 458: 95 - 98

12 Sanger F, Nicklen S, Coulson AR. DNA sequencing with chain-terminating inhibitors. Proc Natl Acad Sci U S A 1977; 74: 5463-5467

13 Molina-Vila MA, Bertran-Alamillo J, Reguart $N$ et al. A sensitive method for detecting EGFR mutations in non-small cell lung cancer samples with few tumor cells. J Thorac Oncol 2008; 3: 1224-1235

14 Do H, Dobrovic A. Limited copy number-high resolution melting (LCNHRM) enables the detection and identification by sequencing of low level mutations in cancer biopsies. Mol Cancer 2009; 8: 82

15 Fassina A, Gazziero A, Zardo D et al. Detection of EGFR and KRAS mutations on trans-thoracic needle aspiration of lung nodules by high resolution melting analysis. J Clin Pathol 2009; 62: 1096-1102 
16 Morinaga R, Okamoto I, Fujita Y et al. Association of epidermal growth factor receptor (EGFR) gene mutations with EGFR amplification in advanced non-small cell lung cancer. Cancer Sci 2008; 99: 2455-2460

17 Marchetti A, Martella C, Felicioni L et al. EGFR mutations in non-smallcell lung cancer: analysis of a large series of cases and development of a rapid and sensitive method for diagnostic screening with potential implications on pharmacologic treatment. J Clin Oncol 2005; 23: 857865

18 Pao W, Ladanyi M. Epidermal growth factor receptor mutation testing in lung cancer: searching for the ideal method. Clin Cancer Res 2007; 13: $4954-4955$

19 Pirker R, Herth FJ, Kerr KM et al. Consensus for EGFR mutation testing in non-small cell lung cancer: results from a European workshop. J Thorac Oncol 2010; 5: 1706-1713

20 Thunnissen E, Kerr KM, Herth FJ et al. The challenge of NSCLC diagnosis and predictive analysis on small samples. Practical approach of a working group. Lung Cancer 2012; 76: 1 - 18

21 Petersen I, Schnabel PA. [What's new in lung pathology: minutes from the Pulmonary Pathology Working Group of the German Society of Pathology]. Pathologe 2011; 32: 351-357

22 Schnabel PA, Petersen I, Junker K. [Current issues in pulmonary pathology: Report of the working group on pulmonary pathology of the German Society of Pathology]. Pathologe 2012; 33 (Suppl.2): 351 - 354

23 Shure D, Fedullo PF. Transbronchial needle aspiration in the diagnosis of submucosal and peribronchial bronchogenic carcinoma. Chest 1985; 88: 49-51

24 Kacar N, Tuksavul F, Edipoglu $O$ et al. Effectiveness of transbronchial needle aspiration in the diagnosis of exophytic endobronchial lesions and submucosal/peribronchial diseases of the lung. Lung Cancer 2005; 50: $221-226$

25 Micames CG, McCrory DC, Pavey DA et al. Endoscopic ultrasound-guided fine-needle aspiration for non-small cell lung cancer staging: A systematic review and metaanalysis. Chest 2007; 131: 539-548

26 Herth FJ, Krasnik M, Kahn N et al. Combined endoscopic-endobronchial ultrasound-guided fine-needle aspiration of mediastinal lymph nodes through a single bronchoscope in 150 patients with suspected lung cancer. Chest 2010; 138: 790 - 794

27 Gellert AR, Rudd RM, Sinha G et al. Fibreoptic bronchoscopy: effect of multiple bronchial biopsies on diagnostic yield in bronchial carcinoma. Thorax 1982; 37: 684-687

28 Popovich JJr, Kvale PA, Eichenhorn MS et al. Diagnostic accuracy of multiple biopsies from flexible fiberoptic bronchoscopy. A comparison of central versus peripheral carcinoma. Am Rev Respir Dis 1982; 125 : $521-523$

29 Hetzel J, Eberhardt R, Herth FJ et al. Cryobiopsy increases the diagnostic yield of endobronchial biopsy: a multicentre trial. Eur Respir J 2012; 39: $685-690$

30 Laurent F, Latrabe V, Vergier B et al. Percutaneous CT-guided biopsy of the lung: comparison between aspiration and automated cutting needles using a coaxial technique. Cardiovasc Intervent Radiol 2000; 23: $266-272$

31 Levine MS, Weiss JM, Harrell JH et al. Transthoracic needle aspiration biopsy following negative fiberoptic bronchoscopy in solitary pulmonary nodules. Chest 1988; 93: 1152 - 1155

32 Fassina A, Corradin M, Zardo D et al. Role and accuracy of rapid on-site evaluation of CT-guided fine needle aspiration cytology of lung nodules. Cytopathology 2011; 22: $306-312$

33 O'Neill AC, McCarthy C, Ridge CA et al. Rapid needle-out patient-rollover time after percutaneous CT-guided transthoracic biopsy of lung nodules: effect on pneumothorax rate. Radiology 2012; 262: 314-319

34 Kazerooni EA, Lim FT, Mikhail A et al. Risk of pneumothorax in CT-guided transthoracic needle aspiration biopsy of the lung. Radiology 1996; 198: $371-375$

35 Khan MF, Straub R, Moghaddam SR et al. Variables affecting the risk of pneumothorax and intrapulmonal hemorrhage in CT-guided transthoracic biopsy. Eur Radiol 2008: 18: 1356-1363
36 Fernandez-Villar A, Gonzalez A, Leiro Vet al. [Effect of different bronchial washing sequences on diagnostic yield in endoscopically visible lung cancer]. Arch Bronconeumol 2006; 42: 278-282

37 van der Drift MA, van der Wilt GJ, Thunnissen FB et al. A prospective study of the timing and cost-effectiveness of bronchial washing during bronchoscopy for pulmonary malignant tumors. Chest 2005; 128: $394-400$

38 Mak VH, Johnston ID, Hetzel MR et al. Value of washings and brushings at fibreoptic bronchoscopy in the diagnosis of lung cancer. Thorax 1990; 45: $373-376$

39 Totsch M, Guzman J, Theegarten D et al. [Bronchoalveolar lavage]. Pathologe 2007; 28: 346-353

40 Thunnissen FB. Sputum examination for early detection of lung cancer J Clin Pathol 2003; 56: 805-810

41 Karahalli E, Yilmaz A, Turker $H$ et al. Usefulness of various diagnostic techniques during fiberoptic bronchoscopy for endoscopically visible lung cancer: should cytologic examinations be performed routinely? Respiration 2001; 68: 611-614

42 Govert JA, Kopita JM, Matchar D et al. Cost-effectiveness of collecting routine cytologic specimens during fiberoptic bronchoscopy for endoscopically visible lung tumor. Chest 1996; 109: 451-456

43 Lee HS, Lee GK, Kim MS et al. Real-time endobronchial ultrasoundguided transbronchial needle aspiration in mediastinal staging of non-small cell lung cancer: how many aspirations per target lymph node station? Chest 2008; 134: 368 - 374

44 Travis WD, Brambilla $E$, Noguchi $M$ et al. International association for the study of lung cancer/american thoracic society/european respiratory society international multidisciplinary classification of lung adenocarcinoma. J Thorac Oncol 2011; 6: 244-285

45 Weihmann J, Weichert C, Petersen I et al. [Evaluation of a cell block method in cytological diagnostics]. Pathologe 2012; 33: 553-559

46 Bubendorf L, Feicher G, Obermann P et al. Zytopathologie, Pathologie Band 9. Berlin, Heidelberg: Springer Verlag; 2011

47 Riaz SP, Luchtenborg M, Jack RH et al. Variation in surgical resection for lung cancer in relation to survival: population-based study in England 2004-2006. Eur J Cancer 2012; 48: 54-60

48 Lau KK, Rathinam S, Waller DA et al. The effects of increased provision of thoracic surgical specialists on the variation in lung cancer resection rate in England. J Thorac Oncol 2013; 8: 68 - 72

49 Savic S, Bihl MP, Bubendorf L. [Non-small cell lung cancer : Subtyping and predictive molecular marker investigations in cytology]. Pathologe 2012; 33: $301-307$

50 Tapia C, Savic S, Bihl M et al. [EGFR mutation analysis in non-small-cell lung cancer: Experience from routine diagnostics]. Pathologe 2009; 30: $384-392$

51 Zlobec I, Raineri I, Schneider $S$ et al. Assessment of mean EGFR gene copy number is a highly reproducible method for evaluating FISH in histological and cytological cancer specimens. Lung Cancer 2010; 68: $192-197$

52 Savic S, Tapia C, Grilli B et al. Comprehensive epidermal growth factor receptor gene analysis from cytological specimens of non-small-cell lung cancers. Br J Cancer 2008; 98: 154-160

53 Bruno P, Mariotta S, Ricci A et al. Reliability of direct sequencing of EGFR: comparison between cytological and histological samples from the same patient. Anticancer Res 2011; 31: 4207-4210

54 Rekhtman N, Brandt SM, Sigel CS et al. Suitability of thoracic cytology for new therapeutic paradigms in non-small cell lung carcinoma: high accuracy of tumor subtyping and feasibility of EGFR and KRAS molecular testing. J Thorac Oncol 2011; 6: 451-458

55 Solomon SB, Zakowski MF, Pao W et al. Core needle lung biopsy specimens: adequacy for EGFR and KRAS mutational analysis. AJR Am J Roentgenol 2010; 194: 266 - 269

56 Chowdhuri SR, Xi L, Pham TH et al. EGFR and KRAS mutation analysis in cytologic samples of lung adenocarcinoma enabled by laser capture microdissection. Mod Pathol 2012; 25: 548-555 